\title{
High Speed Train Rescheduling Based on an Improved Triangle Differential Algorithm
}

\author{
Wenjing Deng ${ }^{1}$ \\ 1.Science and Technology Development Strategy Research Institute Co., Ltd., Chongqing ,401123,China
}

\begin{abstract}
To reduce the total delay time of the high speed trains and produce high quality timetable, this paper establishes a model of the train rescheduling of high-speed railway, describing the related concepts of high speed train rescheduling with matrices。It takes the track number, operation intervals, train dwelling time at stations as constraints, the sum delayed time at all the stations as the operation goal to construct the model of train rescheduling on high speed railway。 Based on the analysis of differential strategy of difference algorithm of, it proposes the differential algorithm for high speed train rescheduling based on triangle differential strategy and presents the steps of the algorithm。 It proves the model effectiveness and the high efficiency and precision of the algorithm with the real data from Beijing-Guangzhou high speed railway。 The method for the high speed train rescheduling based on the differential strategy presented in this paper is reasonable and feasible
\end{abstract}

\author{
KEYWORDS \\ High speed railway \\ Train rescheduling \\ Differential algorithm \\ Triangle strategy
}

\section{INTRODUCTION}

Formulation and adjustment within the railway track train project is the work of Railway Dispatching core, determine the quality of the road section within organizations. Thus, automated railway dispatch command line is focus in recent years. With the continuous improvement of the high-speed rail network, China's railway development has entered a new historical period, how to determine the high-speed train running adjustment programs under the new situation, both to meet the transport needs of passengers and shippers, but also improve the efficiency of the line station, It is a serious problem. Currently, many computing models and intelligent algorithm is applied to solve gradually train planning and adjustment Study up.

For train operation adjustment related scholars in many studies[1]. This work established a linear programming model train shop painting to the most number of trains to run the Objective of the model. This work established a minimum total time late goal Nonlinear Model for Adjusting Train and designed a new particle swarm algorithm to solve it[2]. This work based on the total travel time to establish a minimum of train operation adjustment model and design a two-stage strategy to solve it[3].

Copyright @ 2016 Wenjing Deng

doi: $10.18686 /$ esta.v3i1.4

This is an open-access article distributed under the terms of the Creative Commons Attribution Unported License (http://creativecommons.org/licenses/by-nc/4.0/), which permits unrestricted use, distribution, and reproduction in any medium, provided the original work is properly cited.
Digestion for train running conflicts is NP-hard problem, a columngeneration algorithms based on the adjustment of train operation[4]. Discrete event simulation model based on the establishment of a real-time adjustment of train operation system, and is designed greedy algorithm to solve it[5]. Designed decomposition algorithm Train Operation Adjustment[6] . Work designed the "problem space search for" solving method for train operation adjustment[7]. Work level programming model is designed to run high-speed rail to adjust[8]. Work established a total travel time of the train, running an equalization target a range of services for the target frequency deviation train diagram optimization model design and simulated annealing algorithm[9]. In recent years, with its excellent algorithm difference algorithm performance has been concern, but also applied to solve train operation adjustment and control issues. Work using differential algorithm is built with a train running multiple targets based on control issues[10]. Work to establish a high-speed train running steer a multi-objective optimization model and using differential algorithm to solve it[11] .

Based on the study population classical algorithm, the introduction of multi-strategy and multi-mode addressing population subgroup coevolution mechanism and the improved algorithm is applied to solve the high-speed train operation adjustment of the model to obtain a high-speed train running one kind of adjustment the new solutions. 


\section{HIGH-SPEED RAIL TO RUN ADJUSTMENT MODEL}

When the train is disturbed, resulting train delays, by appropriately adjusting train operation plan, which change in each train station to stop at midnight and run-time interval, the train's final run back up plan diagram, It is the purpose of train operation adjustment. Highspeed railway train operation adjustment is a very complex task, which is directly related to the safety of the railway, the high-speed railway train operation adjustment problems to be studied. In general, the main measures speed train operating conditions are adjusted:

(1) Increase the train stops.

(2) To extend the train stops at the parking division.

(3) Make a reasonable choice of stations, the line station, the train accelerated release.

(4) Organization trains fast, parallel operations, shorten operation time train station.

(5) Full use of the maximum permissible train speed, shorten the Train Running Time.

(6) Other methods: such organizations in the opposite direction of traffic, train merged organization run like.

The implementation of these measures is reflected in the ultimate high-speed train arrival / from the station and the station through.

A total of $\mathrm{N}$ located within the study zone train, $\mathrm{M}$ stations, the socalled car plan refers to a train station in the zone from the start to the division and to send the job nature of each station end station. The train at the station i $k$ plans for a triple [12]. It can be described as:

$P_{i k}=\left\{a_{i k}, p r_{i k}, d_{i k}\right\}, i=1,2, \ldots, N, k=1,2, \ldots, M$

Where, , respectively the train arrival and departure hours after midnight, $P R_{i k}$ show at the train station $\mathrm{k}$ i predetermined type of operation,

$P_{i k}= \begin{cases}1 & \text { Stop } \\ 0 & \text { Pass } \\ -1 & \text { Terminal }\end{cases}$

(2)

So, the actual operating conditions can be made to pass by the train station to characterize each division, which can be made $N \times M$ points program described [12], namely

$O G=\left|\begin{array}{cccc}P_{11} & P_{12} & \ldots & P_{1 M} \\ P_{21} & P_{22} & \ldots & P_{2 M} \\ \ldots & \ldots & P_{i k} & \ldots \\ P_{N 1} & P_{N 2} & \ldots & P_{N M}\end{array}\right|$

Since $P_{i k}$ is a triple, then OG can reach down into the matrix GA, starting and operating matrix GD mark matrix PR:

$G A=\left\{a_{i k}\right\}_{\mathrm{N} \times \mathrm{M}}=\left|\begin{array}{cccc}a_{11} & a_{12} & \ldots & a_{1 M} \\ a_{21} & a_{22} & \ldots & a_{2 M} \\ \ldots & \ldots & a_{i k} & \ldots \\ a_{N 1} & a_{N 2} & \ldots & a_{N M}\end{array}\right|$
Make the following settings:

$$
G A^{0}=\left\{a_{i k}\right\}_{\mathrm{N} \times \mathrm{M}}=\left|\begin{array}{cccc}
a_{11}^{0} & a_{12}^{0} & \ldots & a_{1 M}^{0} \\
a_{21}^{0} & a_{22}^{0} & \ldots & a_{2 M}^{0} \\
\ldots & \ldots & a_{i k}^{o} & \ldots \\
a_{N 1}^{0} & a_{N 2}^{0} & \ldots & a_{N M}^{0}
\end{array}\right|
$$

Established to train working diagram arrival time division matrix.

Lie $S L=\left\{s l_{k}\right\}(k=1,2, \ldots, M)$ represents the number of the station $\mathrm{k}$ to the hairline, $T O=\left\{t_{i k}\right\}(k=1,2, \ldots, M)$ represents technical working time in the train station of $k$. Each has a train entering adjustment earliest access time $h^{k}$, the earliest departure time and $f^{k}$ or at the latest to reach the end zone out of time $g^{k}$, corresponding to the train at a specified period of time[,] inside, it should end in the present operation scheduling zone.

Well, according to the above agreement, the total time at least late train operation adjustment mathematical model as follows:

The objective function

$$
\min z=\sum_{i=1}^{N} \sum_{k=1}^{M} \max \left(a_{i k}-a_{i k}^{0}, 0\right)
$$

Satisfy the constraint

$$
\begin{aligned}
& d_{i k}-a_{i k} \geq p r_{i k} \times t o_{i k} \quad i=1,2, \ldots, N, \quad k=1,2, \ldots, M \\
& a_{i+1 k}>d_{i k} \quad i=1,2, \ldots, N-1, \quad k=1,2, \ldots, M \\
& \left(d_{i+1 k}-d_{i k}\right)^{2}-I^{2} \geq 0 \quad i=1,2, \ldots, N-1, \quad k=1,2, \ldots, M
\end{aligned}
$$

$d_{i k}>a_{i k} \quad i=1,2, \ldots, N, \quad k=1,2, \ldots, M$

$d_{i k}-f^{i} \geq 0 \quad i=1,2, \ldots, N, \quad k=1,2, \ldots, M$

$a_{i k}-h^{i} \geq 0 \quad i=1,2, \ldots, N, k=1,2, \ldots, M$ 


$$
\begin{aligned}
& s l_{k}-N\left(a_{i k}+t_{\text {mon }} \leq d_{i k}\right) \geq 0 \\
& a_{i+1 k}-a_{i k}>\tau_{\text {Depart animal }} \quad i=1,2, \ldots, N-1, k=1,2, \ldots, M \\
& d_{i+1 k}-d_{i k}>\tau_{\text {Depart, anival }} \quad i=1,2, \ldots, N-1, k=1,2, \ldots, M
\end{aligned}
$$

Formula (8) that the train in the station i k downtime must be greater than the time required for technical operations;

The formula (9) represents a train track occupancy start time interval occupied by the end of the evening the same time interval before the train line;

Interval sequential (10) train bound trains must show chronologically sequentially through each section, I was tracking intervals between trains;

Formula (11) represents the same train began to occupy the start time is earlier than the end of the hair line time;

Formula (12) represents all the train departure time is later than the earliest departure time;

Formula (13) represents all the train takes up a start time interval later than the earliest access time;

Formula (14) is sent to the station line capacity constraints, that is, the direction of some kind of train number and the total number of train stations cannot exceed the corresponding number of lines;

where $N\left(a_{i k}+t_{\text {siop }} \leq d_{i k}\right)$ indicates that number the train of arrived at the station but did not leave station $\mathrm{k}$;

(15) (16) cannot be handled at the same time sending and receiving the same direction of the train station, the two vehicles interval should not meet at the same time interval to send $\tau_{\text {Depart, arival }}$ to the hair and not the same interval $\tau_{\text {Arrival, depart }}$.

\section{Differential Evolution Algorithm And Its Improvement Strategy}

\subsection{Basic differential evolution algorithm}

Differential evolution (DE) algorithm is a heuristic algorithm for optimization problems. Essentially, it is a greedy genetic algorithm ensuring quality thinking based on real coding. With the genetic algorithm, differential evolution algorithm consists of mutation and crossover, but compared to genetic algorithm selection operation, differential evolution algorithm uses one-elimination mechanism to update the population. Because of the advantages of differential evolution algorithm in continuous domain optimization problems has been widely used, and lead to boom evolutionary algorithm research. Differential evolution algorithm by the Storm and Price proposed algorithm principle the adoption of individual conduct orientation disturbance, to achieve the purpose of the function value individuals were falling, like other evolutionary algorithms, differential evolution algorithm does not use gradient information function, so the function Derivability even continuity is not required, strong applicability. Meanwhile, the optimization algorithm and the population have in common, but because of differential evolution algorithm to some extent, taking into account the correlation between multiple variables, so compared to the population optimization has a great advantage in the variable in question is coupled.

The basic difference algorithm steps are:

(1) Initial population. L randomly generated chromosomes, wherein each chromosome constituted by a $\mathrm{P}$ genes. Initial population $x_{i}(0), i=1,2, \ldots, L$ where $x_{i}(0)$ constituted by the $\mathrm{P}$ gene for $x_{i 1}(0), x_{i 2}(0), x_{i P}(0)$

(2) Mutation. Using differential strategy chromosome genes mutate. There are several strategies difference, commonly used is randomly selected populations of two chromosomes, and then calculated the difference between the two vectors, multiplied by a scaling factor to obtain a new vector, the vector to be the vector sum of variation, to give the new chromosome.

$v_{i}(k+1)=x_{i 1}(k)+\lambda\left(x_{i 2}(k)-x_{i 3}(k)\right), i \neq r_{1} \neq r_{2} \neq r_{3}$

Where $\lambda$ is the scaling factor. $x_{i}(k)$ represents the $\mathrm{K}$ generation of the i-th individual population.

(3) Cross. In certain probability crossover operation. In order to ensure that genetic variation in the middle of the chromosome to the next generation, it will be a mandatory intermediate gene mutation and chromosomal extraction implants in the next generation of chromosomes.

$\mu_{i j}(k+1)=\left\{\begin{array}{l}v_{i j}(k+1), \text { when } \delta>\text { Random cross } \\ x_{i j}(k), \text { if not }\end{array}\right.$

Where Random cross is a random number between 0 and 1 between.

(4) Select. Differential algorithm uses a greedy algorithm to select individuals into the next generation population.

\subsection{Based on differential evolution algorithm triangle Differential Strategies}

Not difficult to find basic difference algorithm differential strategy is relatively simple, the advantage is in the design of the algorithm is easy to implement, but also to ensure the speed of the algorithm. However, the risk is too simple differential strategy to bring the variability of the chromosome is not sufficiently reflected in the offspring, it could cause results to solve the precocious, trapped in local optimum. Therefore, we design a new differential strategy, take full account of the different characteristics of chromosome mutation, chromosomal better.

This strategy, in the present generation of chromosomes, containing a random selection has mutated chromosome $x_{r 1}(g), x_{r 2}(g) x_{r 3}(g)$ using these three chromosomes, a new generation of chromosomes. The formula is a new generation of chromosomes:

$$
\begin{gathered}
x_{i}(g)=\frac{1}{3}\left[x_{r 1}(g)+x_{r 2}(g)+x_{r 3}(g)\right]+\left(p_{2}-p_{1}\right)\left(x_{r 1}(g)-x_{r 2}(g)\right)+ \\
\left(p_{3}-p_{2}\right)\left(x_{r 2}(g)-x_{r 3}(g)\right)+\left(p_{1}-p_{3}\right)\left(x_{r 3}(g)-x_{r 1}(g)\right) \\
p_{i}=\frac{\left|f\left(x_{r i}(g)\right)\right|}{\left|f\left(x_{r 1}(g)\right)+f\left(x_{r 2}(g)\right)+f\left(x_{r 3}(g)\right)\right|}, i \neq r_{1} \neq r_{2} \neq r_{3}
\end{gathered}
$$


Visible, the new generation of chromosomes update rules take full account of the previous generation of chromosomal characteristics, to avoid the premature phenomenon occurs when the train operation adjustment calculation thus affecting the quality of train operation. adjustment programs.

\subsection{Difference algorithm designed for high-speed rail to run Adjustment Model}

(1) Population size design

Population size design, more appropriate to the size of population is calculated. For this book for train operation adjustment, dimensioning population between 20-50, while ensuring high-speed train running speed adjustment based on iterative calculation to prevent the results of running into local optimum adjustment.

(2) Design of chromosome

The number of trains to $N$, the number of stations it passes is $M$, then there is a path on the way $M-1$ intervals. Because a train two decision variables corresponding to each station at the time of arrival and departure time, the number of decision variables is $2 \times N \times M$. Each variable represents a moment. And because 24 hours is 1440 minutes, so a chromosome as numbers in binary, digital size indicates the number of minutes between the time of 0:00. Such as 8:30 from the 0:00 510 minutes, it expressed as $000,111,111,110$.

(3) Calculate the stop condition

In the high-speed train operation adjustment calculations, iterative design, there are two conditions to stop. By setting one kind of population control algorithm iterations limit cycles. Another goal by controlling the calculated results and the gap between the results of control cycles. In this paper, the second approach, the design is quite simple, when the number of iterations is set more reasonable, it is possible to obtain ideal results.
(4) Calculating step

Designed for train operation adjustment calculation steps are as follows:

First step: Set the calculation results with the allowable difference between the target result;

Step Two: Initial population. Set the particle size of the population is $N_{\text {gen }}$. Each particle position vector from $2 \times N \times M \times N_{\text {gen }}$ variable composition. According train delays situation, set the initial value of each variable;

The third step: Calculate the fitness function of $Z$. The best position of each particle is recorded, and such records fitness function value the smallest chromosome;

The fourth step: Mutation, crossover, select: According to section 3.1 of the mutation, crossover and selection rules chromosome mutation, crossover and selection;

The fifth step: Calculate fitness value, and the difference between the results obtained with the target outcomes, if less than or equal first step in setting the allowable difference, according to the optimal position of the best particle populations, given the high-speed train operation adjustment after each train station arrival and departure time; otherwise, turn the third step.

\section{Calculation examples and analysis}

Select the Beijing-Guangzhou high-speed railway Hengyang South Guangzhou East Train uplink data as the experimental data. As shown in Table 1, between 08:00 and 11:00, Beijing-Guangzhou railway upstream direction, there are G280, G1108, G832, G1002, G1110, G6102, G276, D7802, G1112, G1004, G6104, G542, G1006, G532 and G822 a total of 15 train trips running on the zone. Specific train running times are shown in Table 1.

Table 1 The original timetable between Guangzhounan and Hengyangdong of Beijing-Guangzhou high speed railway from 8:00 to 11:00

\begin{tabular}{|c|c|c|c|c|c|c|c|c|c|c|c|}
\hline & \multicolumn{2}{|c|}{ G280 } & \multicolumn{2}{|c|}{ G1108 } & \multicolumn{3}{|c|}{ G832 } & \multicolumn{2}{|c|}{ G1002 } & \multicolumn{2}{|c|}{ G1110 } \\
\hline & Arrival & Departure & Arrival & Departure & Arrival & & Departure & Arrival & Departure & Arrival & Departure \\
\hline Hengyang East & $8: 41$ & & $8: 52$ & & & $9: 10$ & & $9: 26$ & & $9: 42$ & \\
\hline Moyang Wset & $8: 35$ & $8: 35$ & $8: 47$ & $8: 47$ & & 9:05 & 9:05 & $9: 21$ & $9: 21$ & $9: 36$ & $9: 36$ \\
\hline Bingzhou West & $8: 05$ & $8: 05$ & $8: 17$ & $8: 19$ & & $8: 35$ & $8: 37$ & $8: 50$ & $8: 50$ & 9:05 & 9:05 \\
\hline Shaoguang & & & & & & 8:09 & 8:09 & $8: 24$ & $8: 26$ & $8: 40$ & $8: 40$ \\
\hline Yingde West & & & & & & & & $8: 10$ & $8: 10$ & $8: 25$ & $8: 27$ \\
\hline
\end{tabular}


Arrival Departure Arrival Departure Arrival Departure Arrival Departure Arrival Departure

\begin{tabular}{|c|c|c|c|c|c|c|c|c|c|c|}
\hline Hengyang East & $9: 52$ & & 10:09 & & & & 10:27 & & $10: 59$ & \\
\hline Moyang Wset & $9: 47$ & $9: 47$ & $10: 04$ & 10:04 & & & 10:21 & 10:21 & $10: 54$ & $10: 54$ \\
\hline Bingzhou West & $9: 17$ & $9: 17$ & $9: 35$ & $9: 35$ & $10: 35$ & $10: 35$ & $9: 51$ & 9:51 & $10: 23$ & $10: 25$ \\
\hline Shaoguang & $8: 51$ & $8: 53$ & $9: 11$ & $9: 11$ & 10:06 & $10: 18$ & $9: 26$ & $9: 26$ & $9: 56$ & $9: 56$ \\
\hline Yingde West & $8: 38$ & $8: 38$ & 9:00 & 9:00 & $9: 27$ & $9: 46$ & $9: 11$ & $9: 13$ & $9: 43$ & $9: 43$ \\
\hline Qingyuan & $8: 17$ & $8: 17$ & $8: 39$ & $8: 41$ & 9:03 & 9:10 & $8: 51$ & 8:51 & $9: 22$ & $9: 22$ \\
\hline \multicolumn{11}{|l|}{ Guangzhou } \\
\hline North & $8: 09$ & $8: 09$ & $8: 28$ & $8: 28$ & $8: 39$ & $8: 51$ & $8: 44$ & $8: 44$ & $9: 14$ & $9: 14$ \\
\hline \multicolumn{11}{|l|}{ Guangzhou } \\
\hline South & & $8: 00$ & & $8: 15$ & & $8: 22$ & & $8: 35$ & & 9:05 \\
\hline & \multicolumn{2}{|c|}{ G6104 } & \multicolumn{2}{|l|}{ G542 } & \multicolumn{2}{|l|}{ G1006 } & \multicolumn{2}{|l|}{ G532 } & \multicolumn{2}{|c|}{ G822 } \\
\hline
\end{tabular}

Arrival Departure Arrival Departure Arrival Departure Arrival Departure Arrival Departure

\begin{tabular}{|c|c|c|c|c|c|c|c|c|c|c|}
\hline Hengyang East & $10: 40$ & $10: 42$ & & & & & & & & \\
\hline Moyang Wset & $10: 11$ & $10: 11$ & $10: 43$ & $10: 52$ & & & & & & \\
\hline Bingzhou West & $9: 56$ & 9:56 & $10: 21$ & $10: 23$ & $10: 51$ & $10: 51$ & & & & \\
\hline Shaoguang & $9: 33$ & $9: 33$ & 10:01 & 10:01 & $10: 32$ & $10: 32$ & $10: 47$ & 10:49 & $10: 57$ & $10: 57$ \\
\hline Yingde West & 9:24 & 9:24 & 9:53 & $9: 53$ & $10: 22$ & $10: 24$ & 10:35 & 10:35 & 10:49 & $10: 49$ \\
\hline Qingyuan & & $9: 15$ & & $9: 45$ & & $10: 05$ & & $10: 23$ & & $10: 40$ \\
\hline
\end{tabular}

Suppose railway equipment failures, so one day part of the highspeed train appears late, that G1108 arrives late Chenzhou West 10 points, 13 minutes late arriving at Shaoguan G1002, G1112, G6104, G542, G1006 10 minutes late arriving at the North Canton. Visible, the initial total late time has reached 63 minutes, consider the impact of the spread of train delays, according to section 3.3 Solving the setting procedure, first set the target control value of total delays over 150 minutes, and between results and allow the target result allowable difference of 5 minutes, that is the value of the calculation result is within 155 minutes, to end the cycle calculations.

According to Section 3.3 of the design solution step, the size of the design particle swarm is 20 , then the position vector

$$
2 \times 15 \times 8 \times 20=4800 \text { variable composition. }
$$

Train operation adjustment based on the first three of the improved design of the differential algorithm to calculate the result. Then the results are shown in Table revert to the new train schedule shown in Table 2. Table 2 is indicated as italics moment to moment made after adjustment, the adjusted train diagram shown in Figure 1. 
Table 2 The rescheduled timetable between Guangzhounan and Hengyangdong of Beijing-Guangzhou high speed railway from 8:00 to 11:00

\begin{tabular}{|c|c|c|c|c|c|c|c|c|c|c|}
\hline & \multicolumn{2}{|c|}{ G280 } & \multicolumn{2}{|c|}{ G1108 } & \multicolumn{2}{|c|}{ G832 } & \multicolumn{2}{|c|}{ G1002 } & \multicolumn{2}{|c|}{ G1110 } \\
\hline & Arrival & Departure & Arrival & Departure & Arrival & Departure & Arrival & Departure & Arrival & Departure \\
\hline Hengyang East & \multicolumn{2}{|l|}{$8: 41$} & \multicolumn{2}{|l|}{$8: 54$} & \multicolumn{2}{|l|}{$9: 10$} & \multicolumn{2}{|l|}{$9: 26$} & \multicolumn{2}{|l|}{$9: 42$} \\
\hline Moyang Wset & $8: 35$ & $8: 35$ & $8: 50$ & $8: 50$ & 9:05 & $9: 05$ & $9: 22$ & $9: 22$ & $9: 36$ & $9: 36$ \\
\hline Bingzhou West & $8: 05$ & 8:05 & $8: 27$ & $8: 29$ & $8: 35$ & $8: 37$ & $8: 58$ & $8: 58$ & 9:05 & 9:05 \\
\hline Shaoguang & & & & & 8:09 & 8:09 & $8: 37$ & $8: 39$ & $8: 42$ & $8: 42$ \\
\hline \multirow[t]{3}{*}{ Yingde West } & & & & & & & $8: 10$ & $8: 10$ & $8: 25$ & $8: 27$ \\
\hline & \multicolumn{2}{|c|}{ G6102 } & \multicolumn{2}{|c|}{ G276 } & \multicolumn{2}{|c|}{ D7802 } & \multicolumn{2}{|c|}{ G1112 } & \multicolumn{2}{|c|}{ G1004 } \\
\hline & Arrival & Departure & Arrival & Departure & Arrival & Departure & Arrival & Departure & Arrival & Departure \\
\hline Hengyang East & $9: 52$ & & 10:09 & & & & $10: 27$ & & $10: 59$ & \\
\hline Moyang Wset & $9: 47$ & $9: 47$ & $10: 04$ & $10: 04$ & & & $10: 21$ & $10: 21$ & $10: 54$ & $10: 54$ \\
\hline Bingzhou West & $9: 17$ & $9: 17$ & $9: 35$ & $9: 35$ & $10: 35$ & $10: 35$ & $9: 51$ & $9: 51$ & $10: 23$ & $10: 25$ \\
\hline Shaoguang & $8: 51$ & $8: 53$ & $9: 11$ & $9: 11$ & $10: 06$ & $10: 18$ & $9: 32$ & $9: 32$ & $9: 56$ & $9: 56$ \\
\hline Yingde West & $8: 38$ & $8: 38$ & 9:00 & 9:00 & $9: 27$ & $9: 46$ & $9: 20$ & $9: 22$ & $9: 43$ & $9: 43$ \\
\hline Qingyuan & $8: 17$ & $8: 17$ & $8: 39$ & $8: 41$ & $9: 03$ & $9: 10$ & 9:06 & $9: 06$ & $9: 22$ & $9: 22$ \\
\hline \multicolumn{11}{|l|}{ Guangzhou } \\
\hline North & $8: 09$ & 8:09 & $8: 28$ & $8: 28$ & $8: 39$ & $8: 51$ & $8: 54$ & $8: 54$ & $9: 14$ & $9: 14$ \\
\hline \multicolumn{11}{|l|}{ Guangzhou } \\
\hline \multirow[t]{3}{*}{ South } & & 8:00 & & $8: 15$ & & $8: 22$ & & $8: 35$ & & $9: 05$ \\
\hline & \multicolumn{2}{|c|}{ G6104 } & \multicolumn{2}{|c|}{ G542 } & G1006 & \multicolumn{3}{|c|}{ G532 } & \multicolumn{2}{|c|}{ G822 } \\
\hline & Arrival & Departure & Arrival & Departure & Arrival & Departure & Arrival & Departure & Arrival & Departure \\
\hline Hengyang East & $10: 40$ & $10: 42$ & & & & & & & & \\
\hline Moyang Wset & $10: 15$ & $10: 15$ & $10: 43$ & $10: 52$ & & & & & & \\
\hline Bingzhou West & 10:02 & $10: 02$ & $10: 23$ & $10: 25$ & $10: 56$ & $10: 56$ & & & & \\
\hline Shaoguang & $9: 42$ & $9: 42$ & 10:09 & 10:09 & $10: 40$ & $10: 40$ & $10: 47$ & $10: 49$ & $10: 57$ & $10: 57$ \\
\hline Yingde West & $9: 34$ & $9: 34$ & 10:03 & $10: 03$ & $10: 32$ & $10: 34$ & $10: 37$ & $10: 37$ & $10: 49$ & $10: 49$ \\
\hline Qingyuan & & $9: 15$ & & $9: 45$ & & 10:05 & & $10: 23$ & & $10: 40$ \\
\hline
\end{tabular}


Fig.1 The rescheduled operation chart between Guangzhounan and Hengyangdong of Beijing-Guangzhou high speed railway from 8:00 to $11: 00$

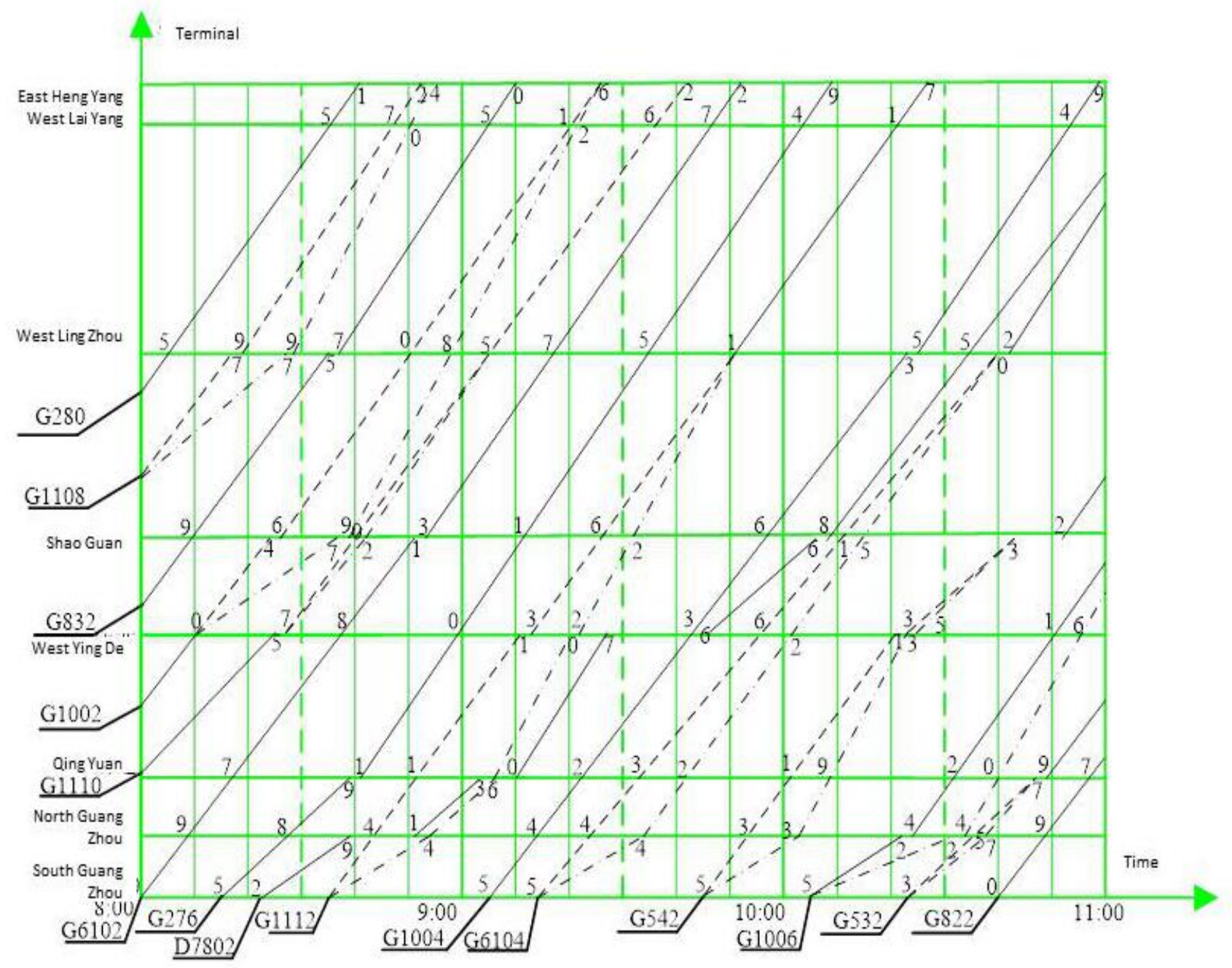

In Figure 1, the dotted lines of the original train plan chart, but due to equipment failure cannot be honored. Dotted line for the new train line adjusted. Found data in Figure 1 and Table 2 from the methods described herein may be designed to give reasonable and feasible train operation plan. Fitness value that is obtained final total late time of 153 minutes, 155 minutes less than the target, and the target 150 minute are just three minutes to reach the algorithm for the calculation accuracy.

Specifically, G1108 since Chenzhou West Railway Station late in Chenzhou and Leiyang west Leiyang Hengyang East interval two points in time, but due to the range of minimum operating hours, when the train arrives at Hengyang East still delayed 2 Minute.

G1002 13 minutes late, and to ensure that the Shaoguan stop $2 \mathrm{~min}$, $8: 39$ to depart from Shaoguan station. At this time, if not late by considering G1110 Shaoguan station, but also to meet the headway between trains, will inevitably result in Shaoguan station is $\mathrm{G} 1110$ G1002 more rows for the same grade, the line cannot G1002 G1110, G1002 so in the end will cause serious delays Therefore, the policy is to be taken at this time $\mathrm{G} 1110$ Shaoguan station arrival time delayed two minutes, G1002 G1110 is still running at the front, and catch the point, making it the West to resume operation in Figure Chenzhou. G1006 and G532 situation between empathy. G1112, G6104 point in time, in the West to resume operation Figure Chenzhou, G542 recovery Figure run in Shaoguan. Visible, models and algorithms designed in this paper, considering the constraints on the basis of train, making the train operation adjustment programs more reasonable. 


\section{Conclusion}

This paper presents a new high-speed railway train operation adjustment method. Conclusions are (1) high-speed railway running adjustment model more complete description of the high-speed train line adjustment work issues. Model test high-speed trains stop time tracking interval, the number of constraints to the hair line, highspeed access to the listed time zone adjustment, not to the hair while at the same time intervals and not sent to the interval time constraints, in order to obtain reasonable speed train adjustment programs laid the foundation. (2) This design strategy triangular differential speed train adjustment algorithm for solving differential adjusting train model established in this paper, a reasonable reduction of the calculated results from the Train Operation Plan Based on. (3) High-speed train operation model and the proposed algorithm is feasible and can provide a reference model and algorithm solutions for train operation scheduling system for construction of a new generation.

\section{References}

1. Cacchiani V, Caprara A, Toth P. Scheduling extra feight trains on railway networks[J]. Transportation Research Part B, 2010,44(2):215-231.

2. Meng X L, Jia L M, Qin Y. Train timetable optimizing and rescheduling based on improved particle swarm algorithm[J], Transportation Research Record, 2010,2197:71-79.
3. Castillo E, Gallego I, Urena J M, et al. Timetabling optimization of a mixed double-and single-tracked railway network[J]. Applied Mathematical Modelling,2011,35(2): 859-878.

4. Min Y H,Park M J, Hong S P, et al. An appraisal of a column-generationbased algorithm for centralized train-conflict resolution on a metropolitan railway network[J]. Transportation Research Part B, 2011,45(2):409-429.

5. Almodóvar M, García-Ródenas R. On-line reschedule optimization for passenger railways in case of emergencies[J]. Computers \& Operations Research, 2013,40(3): 725-736.

6. Lamorgese L, Mannino C. The track formulation for the train dispatching problem[J]. Electronic Notes in Discrete Mathematics, 2013,41:559-566.

7. Albrecht A R, Panton D M, Lee D H. Rescheduling rail networks with maintenance disruptions using problem space search[J]. Computers \& Operations Research,2013,40(3): 703-712.

8. Wang L, Mo W T, Qin Y, et al. Optimization based high-speed railway train rescheduling with speed restriction[J]. Discrete Dynamics in Nature and Society, 2014, Article ID 934369, http://dx.doi.org/10.1155/2014/934369.

9. Huang J, Peng QY. Train diagram optimization of passenger dedicated line based on passenger transport demand in different time[J]. Journal of Railway Science and Engineering, 2012, 9(6): 66-71.

10. HAN Huixin, WU Peng, WU Jie, et al. Coast control of urban train based on multi-objective differential evolution algorithm[J]. Journal of Computer Applications, 2013,33(A02) :286-289.

11. YAN Xihui, CAI Bogen, WU Jie, et al. Research on multi-objective highspeed train operation optimization based on differential evolution[J]. Journal of the China railway Society, 2013,35(9):65-71.

12. JIA Li-min.Fuzzy control and deciding and its application in railway automatization[D]. Beijing: China Academy of Railway Sciences,1991:107-113. 